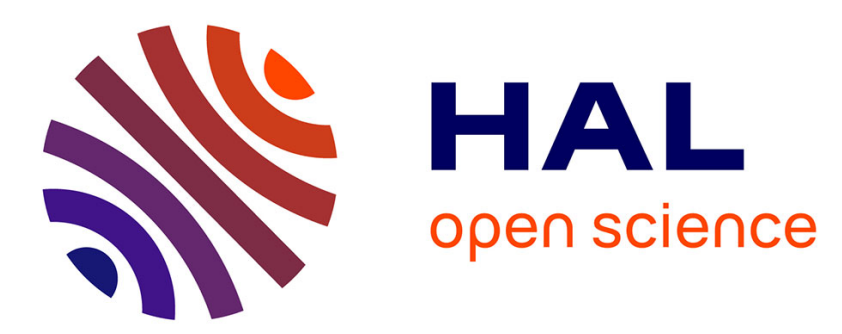

\title{
FIR Filter-Based Online Jerk-Controlled Trajectory Generation
}

\author{
Pierre Besset, Richard Béarée, Olivier Gibaru
}

\section{To cite this version:}

Pierre Besset, Richard Béarée, Olivier Gibaru. FIR Filter-Based Online Jerk-Controlled Trajectory Generation. Industrial Technology (ICIT), 2016 IEEE International Conference, 2016, Unknown, Unknown Region. 10.1109/ICIT.2016.7474730 . hal-01490120

\section{HAL Id: hal-01490120 https://hal-amu.archives-ouvertes.fr/hal-01490120}

Submitted on 4 Jul 2019

HAL is a multi-disciplinary open access archive for the deposit and dissemination of scientific research documents, whether they are published or not. The documents may come from teaching and research institutions in France or abroad, or from public or private research centers.
L'archive ouverte pluridisciplinaire $\mathbf{H A L}$, est destinée au dépôt et à la diffusion de documents scientifiques de niveau recherche, publiés ou non, émanant des établissements d'enseignement et de recherche français ou étrangers, des laboratoires publics ou privés. 


\section{FIR Filter-Based Online Jerk-Controlled Trajectory Generation}

\author{
Pierre Besset \\ Arts et Metiers ParisTech / LSIS \\ 8 Bd Louis XIV, 59000 France \\ Email: pierre.besset@ensam.eu
}

\author{
Richard Béarée \\ Arts et Metiers ParisTech / LSIS \\ Email: richard.bearee@ensam.eu
}

\author{
Olivier Gibaru \\ Arts et Metiers ParisTech / LSIS \\ Email: olivier.gibaru@ensam.eu
}

\begin{abstract}
This paper presents a novel approach to generate online jerk-limited trajectories for multi-DOF robotic systems. Finite Impulse Response filters are used to efficiently turn low computational cost acceleration-limited profiles into jerk-limited profiles. Starting from a new setpoint, e.g. an event given by external sensors, and an arbitrary state of motion, i.e. with nonzero initial velocity and acceleration values, the proposed method can generate different shapes of jerk profile, including timeoptimal and fixed-time jerk-limited trajectories. A new definition of the velocity, acceleration and jerk kinematic limits can be instantaneously taken into account during the motion. Moreover, the very low calculation time (less than 1 microsecond) makes it possible to easily control a multi-DOF system during one control cycle (classically about 1 millisecond), while preserving time for other computer processing. The algorithm is experimentally tested on the new 7-DOF industrial robot KUKA LWR iiwa.
\end{abstract}

\section{INTRODUCTION}

Modern industrial robotic applications often require smooth trajectory generation. Robotic manipulation classically uses smooth profiles, such as jerk-limited trajectories, to avoid acceleration discontinuities, which can induce detrimental vibrations. A significant amount of work have been done to find efficient and elegant solutions to this problem of trajectory planning [1], [2], [3]. As the previous robotic field, service robotics (collaborative) and mobile robotics have to solve the same problem but with online capability. Indeed, in this recent context, robots attempt to instantaneously react to unpredicted events originating from the environment and potentially from the physical human-robot interaction. Therefore, according to the context, the trajectory generation has to verify all or part of the following points:

- Trajectories should be modified during execution time (online capability) depending on external measurements or human commands;

- Trajectories should be time-optimal for productivity reasons;

- The smoothness of the trajectories, at least jerklimited, should depend on the expected behavior (e.g. human motion reproduction or end-point accuracy).

Existing solutions to the problem of time-optimal online trajectory generation can be found in the literature. In [4], authors propose an efficient real-time planner in the operational space, but only the acceleration-limited trajectory (trapezoidal velocity) is considered. In [5], [6], Kröger et al. introduce an elegant framework based on the current state of the system and the final desired state, that provide time-optimal accelerationlimited trajectories. The jerk-limited solution is presented, but not detailed, in their recent works [7]. One notes that this algorithm belonging to the well-known Reflexxes Motion Library [8] is no longer freely available for robotics research. Algorithms based on the analytical recalculation of the timeoptimal third order trajectory parameters can be found in [9], [10]. The computing time of such solutions is about 350 microseconds for one joint trajectory, which can be a significant limitation for multi-axis systems during one control cycle (typically 1 millisecond).

In this work, we propose a faster and more versatile methodology that exploits the property of FIR (Finite Impulse Response) filtering to generate on the fly jerk-limited trajectories, based on initial acceleration-limited trajectories. In addition to the real-time ability, the FIR-based method can be used to generate different jerk pattern (sine squared jerk, damped-jerk [11]) or smoother trajectories (snap-limited, crackle-limited,...) by a simple modification of the filter coefficients or combination of filters.

The next Section presents the methodology adopted for the generation of jerk-limited trajectories. Section III details the procedure to generate online time-optimal jerk-limited trajectories. Section IV shows how the kinematic limits can be changed online. In section V, a fixed-time solution for multiaxis motion synchronization is proposed. Experimental results obtained with a collaborative 7 axis robot are presented in Section VI. Conclusion are drawn in section VII.

\section{TRAJECTORY GENERATION WITH JERK LIMITATION}

The methodology used in this paper is based on the convolution of acceleration-limited trajectories with a FIR filter inducing the final jerk pattern. For instance, convolving a step function of value $e$ with a simple sliding average FIR filter produces a ramp function with ending value $e$ and a slope value of $e / T$ (with $T$ the FIR filter time). Therefore, as illustrated in Figure 1, an acceleration-limited profile may be turned into a jerk-limited profile by FIR filtering. In [12], Biagiotti et Melchiorri generalize this principle by combining FIR average filters in a cascade configuration to obtain smooth trajectories from simple rough step position commands. However, the resulting trajectories cannot be modified online (only zero initial velocity and acceleration conditions could be handled).

In this paper, the subscript $f$ stands for "filtered", i.e. 


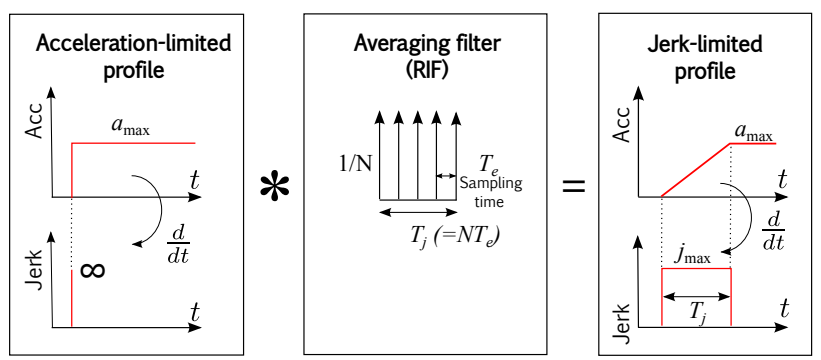

Fig. 1. FIR filtering principle for jerk-limited trajectory synthesis.

denotes the variables of the jerk-limited profile, which is the output of the algorithm. Moreover, for the online trajectory generation, it is assumed that the final velocity and acceleration are zeros, since if no new set-point is given, the goal is reached and the motion stops.

The inputs of the algorithm, presented in Figure 2 are the set-point $q_{e}$, the initial state of the system $\left(q_{0 f}, v_{0 f}, a_{0 f}\right)$, and the kinematics limits $\left(v_{\max }, a_{\max }, d_{\max }, j_{\max }\right)$. The algorithm is divided into two stages. The first one consists in generating an acceleration-limited (AL) trajectory, with is adapted for the following FIR filtering stage. The output of the filtering stage is a jerk-limited (JL) trajectory, which fulfills the requirements in terms of final position, initial state and kinematic limitations on the velocity, acceleration and jerk. As detailed in the remainder of this paper, the adaptation of the initial AL trajectory is based on simple analytical relations. One notes that for the time-optimal solution, this adaptation process can be repeated iteratively according to a decision tree.

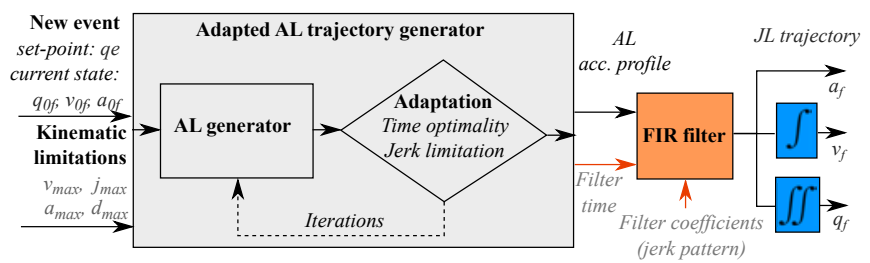

Fig. 2. Proposed online trajectory generation algorithm based on FIR filtering.

\section{A. AL Generator}

The first stage of our methodology consists in generating the time-optimal AL trajectory, which respects the following kinematic constraints and boundary conditions :

$$
\begin{gathered}
\qquad|v(t)| \leq v_{\max } \\
\begin{cases}\text { if } t<T_{a}+T_{v} & |a(t)| \leq a_{\max } \\
\text { else } & |a(t)| \leq d_{\max }\end{cases}
\end{gathered}
$$

The acceleration-limited profile, also known as bang-bang profile, can be build in three phases: first an acceleration phase during which the acceleration is constant: $|a(t)|=a_{\max }$. During the second phase the acceleration is zero, i.e. the maximal velocity has been reached and is constant: $|v(t)|=v_{\max }$. Finally in the third phase comes the deceleration, constant as well: $|a(t)|=d_{\max }$. The durations of these three phases are respectively noted $T_{a}, T_{v}$ and $T_{d}$, as illustrated in Figure 3. The displacement $\Delta q$ is given by relation 2 :

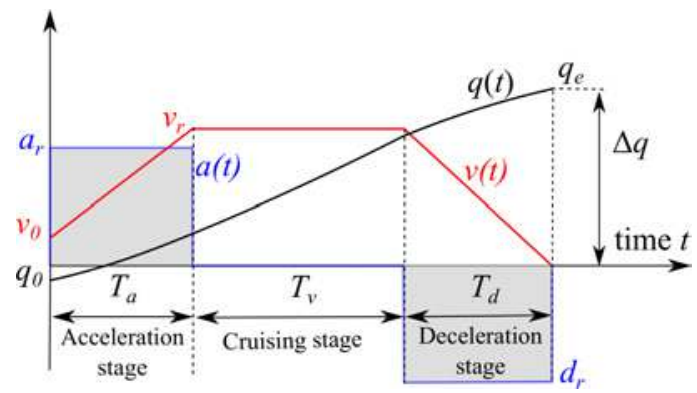

Fig. 3. A classic acceleration-limited profile. $a_{r}, d_{r}$ and $v_{r}$ are the reached acceleration, deceleration and velocity values.

$$
\Delta q=q_{e}-q_{0}=\frac{T_{a}\left(v_{r}+v_{0}\right)}{2}+T_{v} v_{r}+\frac{T_{d} v_{r}}{2}
$$

with:

$$
T_{a}=\frac{v_{r}-v_{0}}{a_{r}} ; T_{d}=\frac{-v_{r}}{d_{r}}
$$

To ensure that the trajectory is time-optimal, we set the acceleration, deceleration, and cruising velocity values at the maximum values:

$$
a_{r}=s \cdot a_{\max } ; \quad d_{r}=-s \cdot d_{\max } ; \quad v_{r}=s \cdot v_{\max },
$$

where $a_{r}, d_{r}$ and $v_{r}$ are the reached acceleration, deceleration and velocity values and $s$ is the sign of the velocity during the cruising phase. In order to take into account a non-zero initial velocity, $s$ can be determined by:

$$
s=\operatorname{sign}\left(q_{e}-q_{s t o p}\right),
$$

where $q_{\text {stop }}$ represents the position reached after the fastest full stop [9]. If the initial velocity $v_{0}$ is greater than the maximum velocity: $s \cdot v_{0}>s \cdot v_{r}$, the velocity has to be reduced to $v_{r}$. In this case, $a_{r}=-s \cdot a_{\max }$ and we have a double deceleration profile where $a_{r}$ and $d_{r}$ have the same sign [9]. Finally, we determine suitable values for $T_{a}, T_{d}$ and $T_{v}$. Let us note $\Delta q_{\text {min }}$ the minimum displacement for which $v_{r}$ is reached, when $T_{v}=$ 0 . If $s \cdot \Delta q \geq s \cdot \Delta q_{\min }$ then the maximal velocity is reached during the motion. Consequently we have:

$$
T_{a}=\frac{v_{r}-v_{0}}{a_{r}} ; T_{v}=\frac{\Delta q-\Delta q_{\min }}{v_{r}} ; T_{d}=\frac{-v_{r}}{d_{r}} .
$$

Otherwise, the maximal velocity is not reached and the velocity profile is wedge-shaped. The maximum reached velocity $v_{r}$ is then updated:

$$
v_{r}=s \cdot \sqrt{\frac{-\frac{v_{0}^{2}}{a r}-2 \Delta q}{\frac{1}{d r}-\frac{1}{a r}}},
$$

and

$$
T_{a}=\max \left(0, \frac{v_{r}-v_{0}}{a_{r}}\right) ; \quad T_{v}=0 ; \quad T_{d}=\max \left(0, \frac{-v_{r}}{d_{r}}\right) .
$$

Finally, the triplet $\left(T_{a}, T_{v}, T_{d}\right)$ defines the time-optimal acceleration-limited profile which respects the kinematic bounds. 


\section{B. Adaptation of the initial AL profile for FIR filtering}

The parameters $\left(q_{0}, v_{0}\right)$ used to generate the initial $\mathrm{AL}$ profile have to be adjusted according to the initial state $\left(q_{0 f}, v_{0 f}, a_{0 f}\right)$. The relationships between the parameters of the non-filtered and filtered profiles are given by equations (7) and (8).

$$
\begin{gathered}
v_{0}=v_{0 f}+\frac{T_{j} a_{0 f}}{2}, \\
q_{0}=q_{0 f}+T_{j} v_{0 f}+\frac{T_{j}^{2} a_{0 f}}{3}+\frac{T_{j}}{2}\left(T_{a} a_{r}+T_{d} d_{r}\right),
\end{gathered}
$$

with $T_{j}$ the filter time, as shown in Figure 4.

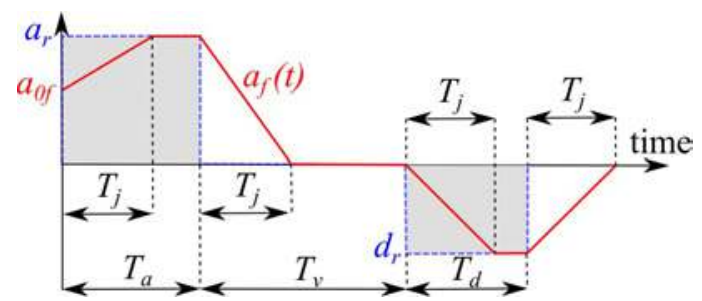

Fig. 4. The initial acceleration-limited trajectory (blue dashed line) is filtered to give a jerk-limited profile (solid red line).

Once the AL profile is generated, the test $T_{v}>T j$ should be checked. If $T_{v}$ is less than $T_{j}$, an adaptation of the $\mathrm{AL}$ profile is required to keep the jerk of the JL profile from exceeding its limits, as shown on Figure 5. Such an adaptation consists in re-generating the AL profile with a lower value of $v_{r}$ which satisfies $T_{v}=T_{j}$ and (5).
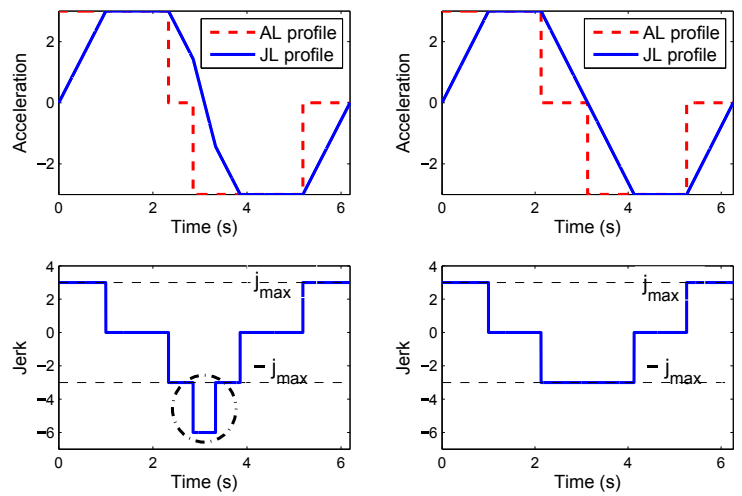

Fig. 5. Example of an adaptation: Type II (see Fig. 11). To the left: nonadapted profile, $T_{v}<T_{j}$. To the right, adapted profile, $T_{v}=T_{j}$ and $\left|v_{r}\right|<$ $v_{\max }$.

\section{FIR Filtering and Jerk pattern}

Starting from the previous AL profile, a JL trajectory can be obtained using a FIR moving average filter, as shown on Figure 4 . The length of the filter $T_{j}$ is classically chosen to reach the maximum value of the jerk:

$T_{j}=\max \left(\left|a_{r}-a_{0 f}\right|, a_{\max }, d_{\max }\right) / j_{\max }$. In order to respect the desired initial acceleration of the profile, the coefficients of the filter should be initialized at $a_{0 f}$. One notes that the resulting $\mathrm{JL}$ trajectory is not time-optimal for all kinds of motion because the jerk time is fixed instead of the maximum jerk value. Other jerk patterns can be generated by changing the coefficients of the filters. For instance, jerk patterns can be used to reduce residual vibrations in the system as described in [11]. Figure 6 presents two examples of trajectories generated using Damped-Jerk and sine squared Jerk patterns.
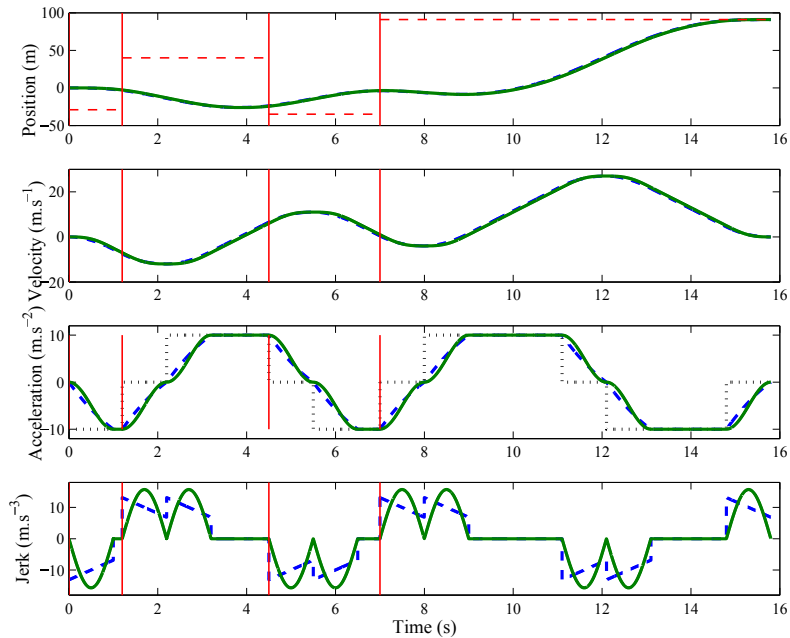

Fig. 6. Example of trajectories with different jerk patterns: Damped-Jerk (dashed blue line) and sine squared Jerk (solid green line).

\section{TIME-OPTIMAL JL TRAJECTORY}

Many industrial applications require time-optimal trajectories for productivity reasons. To ensure the time-optimality, the maximum jerk value should be fixed instead of the filter time. Therefore the filter time should be changed while filtering to satisfy the following relationships:

$$
T_{j a}=\frac{\left|a_{r}-a_{0 f}\right|}{j_{\max }} ; T_{j v}=\frac{\left|a_{r}\right|}{j_{\max }} ; T_{j d}=\frac{\left|d_{r}\right|}{j_{\max }},
$$

where $T_{j a}, T_{j v}$ and $T_{j d}$ are the durations of the constant jerk stages, as shown in Figure 7.

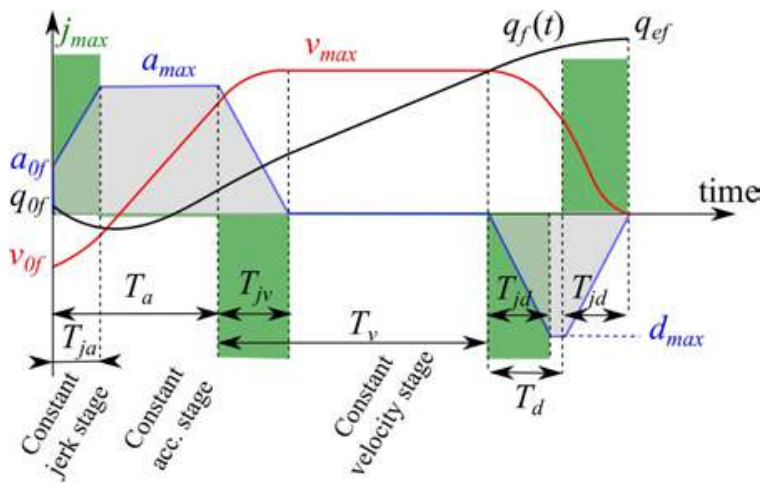

Fig. 7. Time-optimal jerk-limited profile with non-zero initial conditions, each derivative saturates at its bound.

Before generating the $\mathrm{AL}$ profile, the sign $s$ has to be determined, i.e. the fastest full-stop position $q_{\text {stop }}$ which can be reached under the kinematic bounds, as stated in (4). According to the state of the system, the acceleration profile leading to the fastest full-stop of the system can take different 
shapes, as Figure 8 shows. If $q_{\text {stop }}=q_{e}$ then the full-stop profile should be kept as a result with no further calculation. If $\operatorname{sign}\left(a_{0 f}\right) \neq \operatorname{sign}\left(a_{r}\right)$, the sign $s$ should be reevaluated at

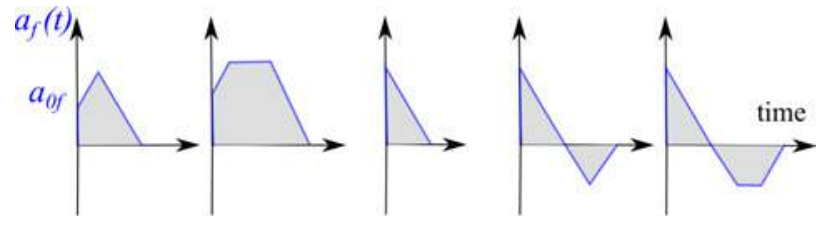

Fig. 8. Examples of fullstops acceleration profiles.

$t=\left|a_{0 f} / j_{\max }\right|$. In the case of a sign change, the trajectory is recalculated from the time $T_{p}$ where $s$ changes. Figure 9 shows such a trajectory.

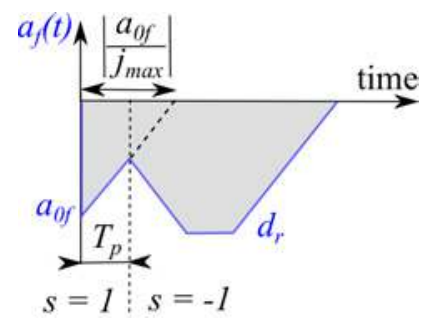

Fig. 9. Example of an acceleration profile with a sign change at $t=T_{p}$.

\section{A. Adaptation of the AL profile}

The relationships between $\left(q_{0}, v_{0}\right)$ and $\left(q_{0 f}, v_{0 f}, a_{0 f}\right)$ given by (7) and (8) cannot be used for time-optimal generation since the length of the filter is not constant in the general case. Equations (10) and (11) are derived to take this difference into account.

$$
\begin{gathered}
v_{0}=s \cdot \frac{a_{r} a_{0 f}}{j_{\max }}-s \cdot \frac{a_{0 f}^{2}}{2 j_{\max }}+v_{0 f}, \\
q_{0}=a_{r}\left(\frac{s \cdot v_{0 f}}{2 j_{\max }}+\frac{a_{0 f}^{2}}{4 j_{\text {max }}^{2}}\right)+q_{0 f}-\frac{a_{0 f}^{3}}{6 j_{\max }^{2}}-s\left(a_{r}+d_{r}\right) \frac{v_{r}}{2 j_{\max }} .
\end{gathered}
$$

In addition to the adaptation previously described in II-B, which makes sure that the jerk bound is respected, the AL profile should also be checked to make sure that the solution given after filtering is time-optimal. If $T_{j a}>T_{a}$ or $T_{j d}>T_{d}$, then the acceleration profile after filtering saturates at a value less than its bound, respectively $a_{\max }$ or $d_{\max }$, as illustrated on Figure 10. In that case, the initial AL profile should be adapted in order to produce the desired time-optimal jerklimited trajectory. Figure 11 presents the decision table used to select the adaptation to be applied to the AL profile. As an example, if the initial AL profile exhibits $T_{d}<T_{j d}$ and $T_{v}>T_{j v}$, a Type III-b adaptation is needed. In that case, the deceleration stage does not saturate, i.e. $\left|d_{r}\right|<d_{\max }$. Therefore

$$
T_{d}=T_{j d} \Rightarrow \frac{-v_{r}}{d_{r}}=\frac{-s \cdot d_{r}}{j_{\max }}
$$

hence:

$$
d_{r}=-s \sqrt{s j_{\max } v_{r}} .
$$

Once the AL profile is adapted, it should be re-checked for a possible second adaptation, as shown in Figure 12.
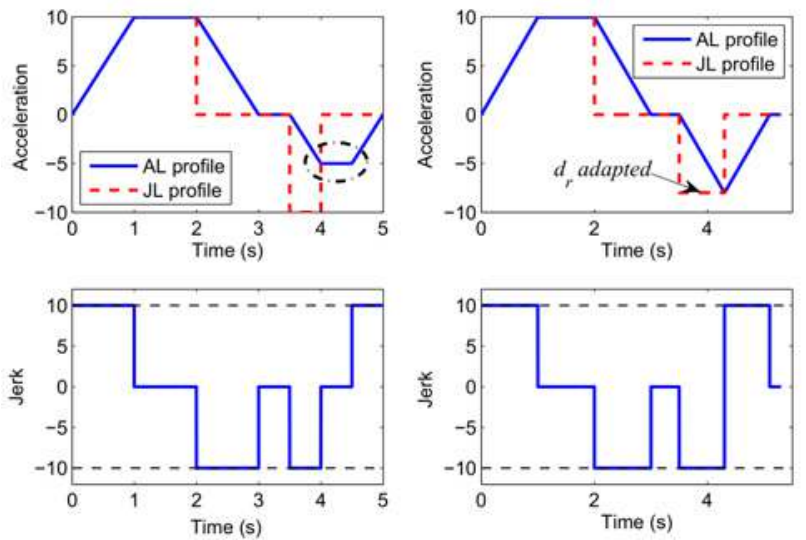

Fig. 10. Example of an adaptation: Type III-b (see Fig. 11). To the left: non-adapted profile, $T_{d}<T_{j d}$. To the right, adapted profile, $T_{d}=T_{j d}$ and $\left|d_{r}\right|<d_{\max }$.

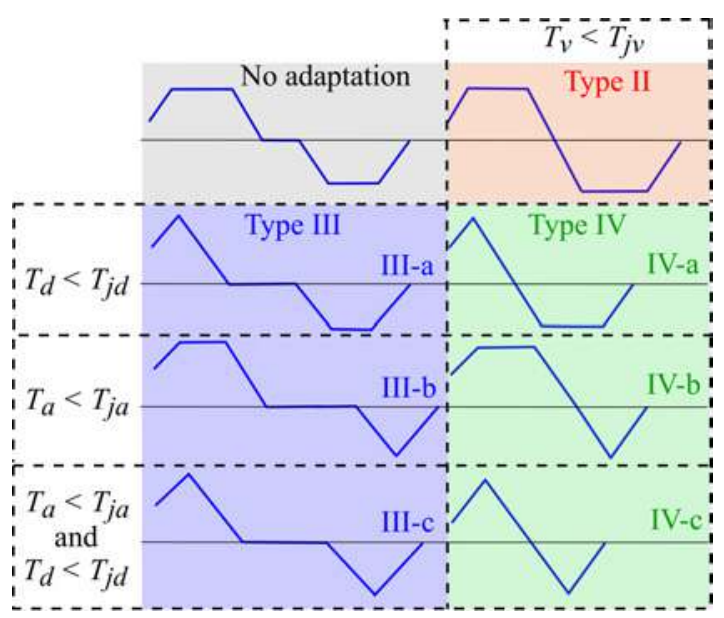

Fig. 11. Decision table for the selection of the adaptation of the AL profile.

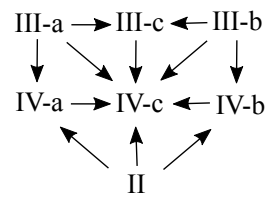

Fig. 12. Decision tree which shows the possible successive adaptations.

\section{B. Filtering}

In order to keep the jerk at its maximum value, the length of the filter is dynamically switched according to relation (9). The trajectory starts at $t=0$ with a filter initialized at $a_{0 \mathrm{f}}$ with a length $T_{j a}$. Then the filter is changed at $t=T_{a}$ to a length $T_{j v}$, initialized at $a_{r}$. Finally at $t=T_{a}+T_{v}$ the length of the filter is set to $T_{j d}$.

\section{ONLINE CONSTRAINTS CHANGE}

Many recent robotic applications require online adaptation of the kinematic bounds of the trajectory. For instance, the detection of human presence in the workspace of the robot can enable a "smooth" mode in which the kinematic limits are lowered, or the jerk pattern modified. In the case where the velocity or acceleration limit is lowered during the motion, a 
transition stage may be required. If the initial acceleration and velocity are such that the new velocity limits are or will be exceeded, i.e. if:

$$
s \cdot\left(v_{0 f}+\frac{a_{0 f}}{2} \cdot\left|\frac{a_{0 f}}{j_{\max }}\right|\right)>s \cdot v_{\max },
$$

then the deceleration stage, noted $a_{d s}(t)$, should bring the velocity back into its news bounds as soon as possible. Let us note the duration of this stage $T_{d s}$, as shown in Figure 13. We want:

$$
v_{0 f}+\int_{t_{0}}^{T_{d s}} a_{d s}(t) d t=s \cdot v_{\max } .
$$

For the fastest solution, we saturate the jerk:

$$
v_{d s}(t)=-s \cdot \frac{j_{\max } t^{2}}{2}+a_{0 f} t+v_{0 f} .
$$

We want $v_{d s}\left(T_{d s}\right)=s \cdot v_{\max }$, hence

$$
-s \cdot \frac{j_{\max }}{2} T_{d s}^{2}+a_{0 f} T_{d s}+v_{0 f}-v_{\max }=0,
$$

if $T_{d s}>\left|\frac{-s \cdot a_{\max }-a_{0 f}}{j_{\max }}\right|$ then $a_{d s}$ should saturate at $-s \cdot a_{\max }$.

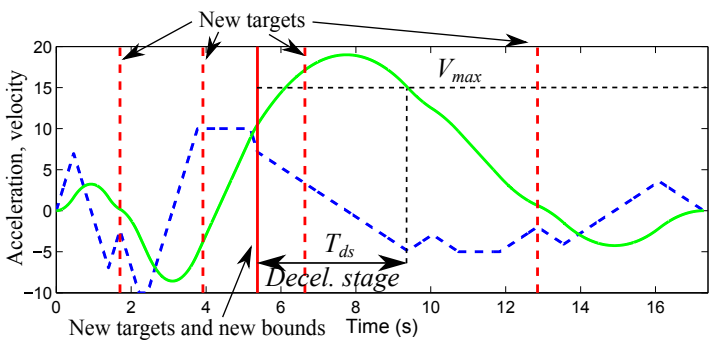

Fig. 13. Example of a trajectory with a deceleration stage. Dashed blue line: acceleration. Full green line: velocity. The kinematic bounds are lowered at $t=5.3 \mathrm{~s}$ (vertical full line). A deceleration stage ensures that the velocity respects its new limit.

\section{Multi-Dimensional CASE / Axis SYNCHRONIZATION}

In the general case, several Degrees-Of-Freedom (DOF) are controlled simultaneously to reach a targeted position. Classically, it is wished that the motion of each DOF ends at the same time. Consequently, for a motion involving $n$ DOFs, with $T_{i}$ the duration of the time-optimal trajectory for the $i$-th DOF, $n-1$ trajectories have to be adjusted to the fixed time constraint $T_{f i x}$ given by the slowest DOF

$$
T_{f i x}=\max _{i} T_{i}
$$

This adjustment can be done in several, very different ways. Here, we propose to take benefits from the filtering strategy to easily compute the fixed-time solution for the JL profile. Indeed, without consideration of time-optimality, if an $\mathrm{AL}$ profile is directly convolved with a FIR filter of time duration $T_{j}$, the resulting profile is $T_{j}$ seconds longer than the initial AL profile.

Noting $\tau_{f i x}$ the fixed time duration of the AL profile, we have the relation $\tau_{f i x}=T_{f i x}-T_{j}$. The AL profile used here allows arbitrary initial velocities and the fixed-time solution for such a profile can be simply calculated. Considering the rescaling of the whole time evolution with the parameter $\alpha=$ $\tau_{\text {fix }} / \tau_{i}$ :

$$
\tau_{a}=\alpha T_{a} ; \tau_{v}=\alpha T_{v} ; \tau_{d}=\alpha T_{d} .
$$

The parameters to be determined are $a_{r}$ and $d_{r}$, respectively the reached acceleration and deceleration. From (2) and (3) it is straightforward to derive the relationships (16) and (17):

$$
\begin{gathered}
a_{r}=-\frac{q_{0}+\tau_{a} \cdot v_{0}+\tau_{d} \cdot v_{0} / 2+\tau_{v} \cdot v_{0}-q_{e}}{\tau_{a}^{2} / 2+\tau_{a} \cdot \tau_{d} / 2+\tau_{a} \cdot \tau_{v}}, \\
d_{r}=-\frac{v_{0}+\tau_{a} \cdot a_{r}}{\tau_{d}},
\end{gathered}
$$

where $q_{0}$ and $v_{0}$ are the ones used to generate the initial trajectory, i.e. the ones given by the relationships (7) and (8). Thus the axis synchronization problem for JL trajectories is solved with a set of simple equations. Since no iterative root-finding is required, these equations are almost instantaneous to evaluate. Equations (10) and (11) stand for trajectories with a jerk profile saturated at a given value $j_{\max }$. Since synchronized trajectories do not respect that condition anymore, another method has to be developed. In order to minimize the computation time, it is wished to avoid iterative resolutions of equations. By setting the jerk at its maximum value for the first acceleration phase and preserving the length of the filter for the deceleration phase $T_{j d}$, as shown on Figure 14, the problem is simplified. $T_{j a}$ and $T_{j v}$ can be expressed as

$$
T_{j a}=s \cdot \frac{a_{r}-a_{0 f}}{j_{\max }}, T_{j v}=s \cdot \frac{a_{r}}{j_{\max }} .
$$

The unknown parameters are the reached accelerations and deceleration $a_{r}$ and $d_{r}$, which are the solution of the system (19):

$$
\left\{\begin{array}{l}
A \cdot a_{r}^{2}+B \cdot a_{r}+C=0 \\
d_{r}=-\frac{v_{0 f}+\left(a_{0 f}+a_{r}\right) * T_{j a} / 2+a_{r} *\left(\tau_{a}-T_{j a}\right)+a_{r} * T_{j v} / 2}{\tau_{d}}
\end{array}\right.
$$

with

$$
\begin{aligned}
& A=-\frac{a_{0 f}+j_{\max } s \tau_{a}}{2 j_{\text {max }}^{2}} \\
& B=\frac{\tau_{a}\left(T_{j d}+\tau_{a}+\tau_{d}+2 \tau_{v}\right)}{2}+\frac{a_{0 f}^{2}}{2 j_{\max }^{2}}+\frac{a_{0 f} s\left(T_{j d}+2 \tau_{a}+\tau_{d}+2 \tau_{v}\right)}{2 j_{\max }} \\
& C=q_{0 f}-q_{e f}+v_{0 f}\left(\frac{T_{j d}}{2}+\tau_{a}+\frac{\tau_{d}}{2}+\tau_{v}\right)-\frac{a_{0 f}^{3}}{6 j_{\max }^{2}} \\
& -s \cdot \frac{a_{0 f}^{2}\left(T_{j d}+2 \tau_{a}+\tau_{d}+2 \tau_{v}\right)}{4 j_{\max }}
\end{aligned}
$$

This system gives an exact solution while $s \cdot a_{0 f} \leq s \cdot a_{r}$. Otherwise, $T_{j a}=-s \cdot \frac{a_{r}-a_{0 f}}{j_{\max }}$, thus the expression of $A, B$ and $C$ changes. This approach is very fast, since it only requires to find the roots of a second order polynomial function.

Figure 15 shows an example of synchronized jerk-limited trajectories.

\section{RESULTS}

The algorithm has been tested for all types of configurations to validate its results. In any case the algorithm generates the solution in less than 1 microsecond on a regular laptop i7 / $2.7 \mathrm{GHz}$ running with Windows 7 as operating system. At the best of the authors knowledge this is the fastest time-optimal jerk-limited trajectory generator. A typical control cycle time being $1 \mathrm{~ms}$, our algorithm can easily handle any multi-axis 


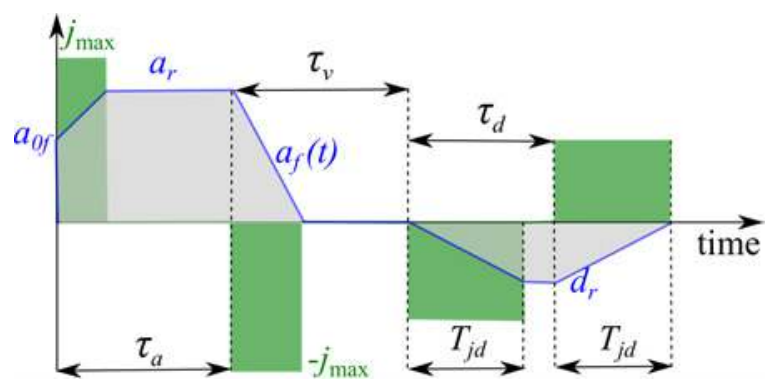

Fig. 14. The approach chosen to deal with joint synchronization is to constrain the value of the jerk for the first acceleration stage and the filter length for the second one.

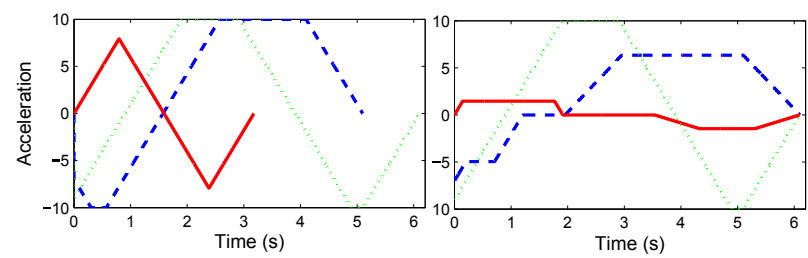

Fig. 15. Synchronization of time-optimal trajectories: initial trajectories to the left, synchronized trajectories to the right.

system and save a significant amount of time to the controller for other tasks, e.g. monitoring, obstacle avoidance or any data processing. Besides, such a small calculation time enable instantaneous reactions to unforeseen events, as introduced in [5].

The algorithm has been tested with a 7 DOF KUKA leightweigth LBR IIWA. The Fast Robot Interface (FRI) of the KUKA Sunrise.Connectivity collection of open interfaces was used for real time communication between the robot controller and an external computer, with a rate of $1 \mathrm{~ms}$. Figures 16 and 17 show samples of experimental results with respectively synchronized and not synchronized joints. Dynamic new target references were randomly generated and the new trajectories updated online, with some changes in the kinematic limits.
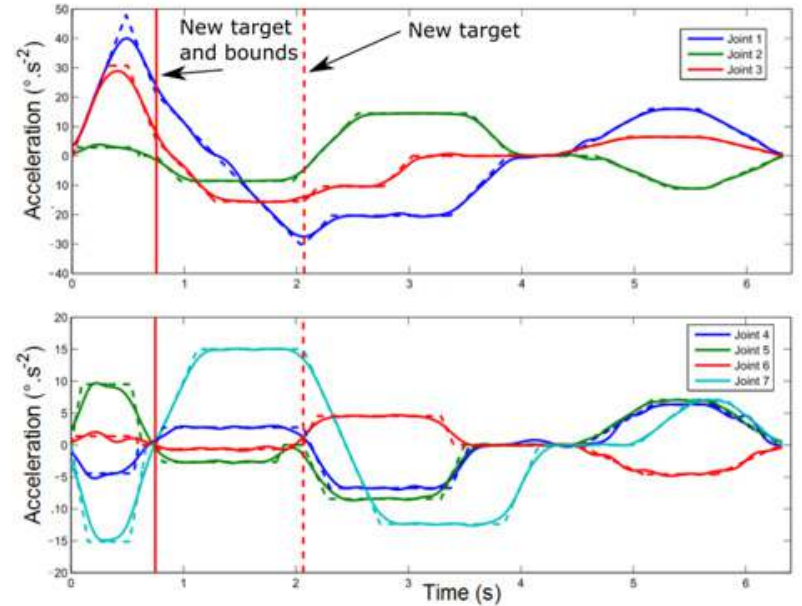

Fig. 16. Experimental results for synchronized trajectories (7 joints). New targets are given on vertical lines. $j_{\max }$ is lowered at the full vertical line Dashed lines: reference acceleration, full lines: measured acceleration. Top graph: joints 1 to 3 ; bottom graph: joints 4 to 7 .

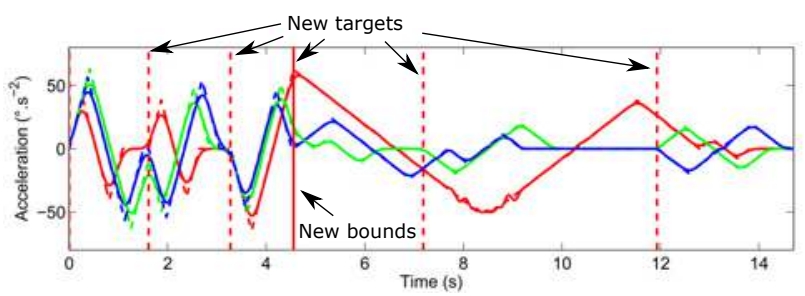

Fig. 17. Experimental results for non-synchronized trajectories (only three joints shown). New targets are given on vertical lines. $j_{\max }$ is lowered at the vertical full line. Dashed lines: reference acceleration, full lines: measured acceleration.

\section{CONCLUSION}

A method to generate online jerk-limited trajectories based on FIR filtering of adapted acceleration-limited profiles is presented in this paper. This generator handles non-zero velocity and acceleration as well as non-symmetric acceleration bounds. Time-optimal and time-fixed solutions for multi-axis synchronization are also proposed. The proposed method generates time-optimal trajectories in less than one microsecond on a regular laptop.

\section{REFERENCES}

[1] K. Erkorkmaz and Y. Altintas, Highspeed cnc system design. Part I: Jerk limited trajectory generation and quintic spline interpolation, I.J. Machine Tools \& Manufacture, vol. 41, pp. 1323-1345, 2001.

[2] S.Y. Jeong, Y.J. Choi, P. Park, and S.G. Choi, Jerk limited velocity profile generation for high speed industrial robot trajectories, in Proc. of the IFAC World Congress, Praha, Czech Republic , vol. 16(1), pp. 1362-1368, 2005.

[3] A. Olabi, R. Béarée, O. Gibaru, and M. Damak, Feedrate planning for machining with industrial six-axis robots, Control Engineering Practice, vol.18(5), pp. 471-482, 2010.

[4] R. Béarée, A. Olabi, Real-Time Planner in the Operational Space for the Automatic Handling of Kinematic Constraints, IEEE Trans. Robot. Autom., 11(3) : 730-739, 2014.

[5] T. Kröger, A. Tomiczek, and F. M. Wahl, Towards on-line trajectory computation, in Proc. of the IEEE/RSJ International Conference on Intelligent Robots and Systems, Beijing, China, 2006, pp. 736-741.

[6] T. Kröger and F. M. Wahl, On-line trajectory generation: Basic concepts for instantaneous reactions to unforeseen events, IEEE Trans. on Robotics, vol. 26, no. 1, pp. 94-111, 2010.

[7] T. Kröger, Opening the Door to New Sensor-Based Robot Applications - The Reflexxes Motion Libraries, In Proc. of the IEEE International Conference on Robotics and Automation, Shanghai, China, May 2011.

[8] Reflexxes Motion Library. http://www.reflexxes.ws.

[9] R. Haschke, E. Weitnauer and H. Ritter, On-line planning of timeoptimal, jerk-limited trajectories, in Proc. of the IEEE/RSJ International Conference on Intelligent Robots and Systems, Nice, France, 22-26 Sept. 2008, pp.3248-3253.

[10] F. Ramos, M. Gajamohan, N. Huebel and R. D'Andrea, Time-optimal online trajectory generator for robotic manipulators, report of Institute for Dynamic Systems and Control, 2013.

[11] R. Béarée, New Damped-Jerk trajectory for vibration reduction, Control Engineering Practice, vol.28(14), pp. 112-120, 2014.

[12] L. Biagiotti and C. Melchiorri, FIR filters for online trajectory planning with time and frequency-domain specifications, Control Engineering Practice, vol.20(12), pp. 1385-1399, 2012.

[13] R. Béarée, A. Olabi, Dissociated jerk-limited trajectory applied to time-varying vibration reduction, Robotics and Computer-Integrated Manufacturing, 29(3) : 444-453, 2013. 